\title{
INDUÇÃO DO ENRAIZAMENTO DE ESTACAS DE ARATICUM-DE-PORCO PELA APLICAÇÃO DE FITORREGULADORES
}

\section{ROOTING OF ARATICUM-DE-PORCO STEM CUTTINGS WITH PLANT GROWTH REGULATORS}

\author{
Lausanne Soraya PINTO ${ }^{1}$ \\ Katia Christina ZUFFELLATO-RIBAS ${ }^{2}$ \\ Antonio Aparecido CARPANEZZI ${ }^{3}$ \\ Fernando Rodrigues TAVARES ${ }^{3}$ \\ Henrique Soares KOEHLER ${ }^{4}$
}

\begin{abstract}
RESUMO
Rollinia rugulosa Schl. (Annonaceae) é uma espécie nativa brasileira que produz frutos comestíveis atraindo a fauna local, sendo indicada para reflorestamentos ambientais. A produção de mudas via sementes apresenta dificuldades. Para investigar sua propagação via estaquia, foram realizadas coletas de ramos semilenhosos de árvores nativas adultas localizadas em Colombo - PR, nas quatro estações do ano, a partir de março de 2000. As estacas foram submetidas aos seguintes tratamentos com ácido indol butírico (AIB) e ácido naftaleno acético (ANA): $2000 \mathrm{mg} \cdot \mathrm{L}^{-1} \mathrm{AlB}, 4000 \mathrm{mg} \cdot \mathrm{L}^{-1} \mathrm{AlB}, 6000 \mathrm{mg} \cdot \mathrm{L}^{-1} \mathrm{AlB}, 2000 \mathrm{mg} \cdot \mathrm{L}^{-1} \mathrm{ANA}, 4000 \mathrm{mg} \cdot \mathrm{L}^{-1} \mathrm{ANA}$, $6000 \mathrm{mg} \cdot \mathrm{L}^{-1} \mathrm{ANA}, 2000 \mathrm{mg} \cdot \mathrm{L}^{-1} \mathrm{AlB}+2000 \mathrm{mg} \cdot \mathrm{L}^{-1} \mathrm{ANA}$, além de $5000 \mathrm{mg} \cdot \mathrm{L}^{-1} \mathrm{ANA}$ (Raizon $05 \AA$ ) na forma de talco e da testemunha (água destilada). Estacas coletadas no outono e no inverno não enraizaram. A porcentagem máxima de enraizamento (4\%) foi obtida nos tratamentos com $6000 \mathrm{mg} . \mathrm{L}^{-1} \mathrm{AlB}$ e $2000 \mathrm{mg} \cdot \mathrm{L}^{-1} \mathrm{AlB}+2000 \mathrm{mg} \cdot \mathrm{L}^{-1} \mathrm{ANA}$, na primavera, indicando que Rollinia rugulosa apresenta um baixíssimo potencial de enraizamento nas épocas e condições testadas.
\end{abstract}

Palavras-chave: Rollinia rugulosa, Annonaceae, auxinas, estaquia.

\begin{abstract}
Rollinia rugulosa Schl. (Annonaceae) is a native tree species suitable for ecosystem rehabilitation. This species produces edible fruits, that attract the local fauna. However, it presents a low rate of seed germination. To investigate the asexual reproduction, stem cuttings from native plants were collected in Colombo, State of Paraná, in the four seasons, beggining in march/2000. The

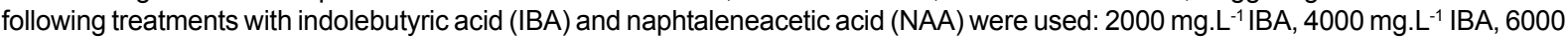
mg.L.-1 IBA, $2000 \mathrm{mg} \cdot \mathrm{L}^{-1} \mathrm{NAA}, 4000 \mathrm{mg} \cdot \mathrm{L}^{-1} \mathrm{NAA}, 6000 \mathrm{mg} \cdot \mathrm{L}^{-1} \mathrm{NAA}, 2000 \mathrm{mg} \cdot \mathrm{L}^{-1} \mathrm{IBA}+2000 \mathrm{mg} \cdot \mathrm{L}^{-1} \mathrm{NAA}$, and $5000 \mathrm{mg} \cdot \mathrm{L}^{-1} \mathrm{NAA}$ (Raizon $05\left(\right.$ ) in powder and the control (water). Cuttings collected during the fall and winter did not develop roots. Treatments using $6000 \mathrm{mg} \cdot \mathrm{L}^{-}$ ${ }^{1}$ IBA and $2000 \mathrm{mg} \cdot \mathrm{L}^{-1}$ IBA $+2000 \mathrm{mg} \cdot \mathrm{L}^{-1} \mathrm{NAA}$ showed the best results, during the spring, with $4 \%$ of root formation, for both treatments. Rollinia rugulosa showed a very low rate of rooting during the seasons for the tested conditions.

Key-words: Rollinia rugulosa, Annonaceae, auxins, cutting.
\end{abstract}

\footnotetext{
${ }^{1}$ Eng. Florestal, Universidade Federal do Paraná (UFPR), Curitiba, PR;

${ }^{2}$ Bióloga, Dra., Depto. Botânica, Setor de Ciências Biológicas, Universidade Federal do Paraná (UFPR), Profa. Adjunta. Caixa Postal 19031, CEP 81531-970, Curitiba, PR, kazu@ufpr.br:

${ }^{3}$ Eng. Florestal, Dr., Pesquisador Embrapa Florestas, Colombo, PR;

${ }^{4}$ Eng. Florestal, Dr., Depto. Fitotecnia e Fitossanitarismo, Setor de Ciências Agrárias, Universidade Federal do Paraná (UFPR), Prof. Adjunto.
} 
PINTO, L.S. et al. Indução do enraizamento de estacas...

\section{INTRODUÇÃO}

O araticum-de-porco (Rollinia rugulosa Schl., Annonaceae) é uma árvore nativa de até $12 \mathrm{~m}$ de altura e $40 \mathrm{~cm}$ de diâmetro, nativa no Brasil (desde Minas Gerais até o Rio Grande do Sul), Argentina, Bolívia, Paraguai e Peru. Na Região Sul do Brasil ela ocorre em vários biomas, como Florestas Ombrófilas Densa e Mista (nas quais é mais comum), Florestas Estacionais e nos capões da Savana (REITZ et al,. 1983; ZÁCHIA,1994).

Além de ser considerada uma espécie ornamental, o araticum-de-porco produz frutos comestíveis pelo homem, de sabor levemente ácido, e madeira de reduzida utilidade; a casca fornece fibras que podem ser utilizadas na fabricação de cordas (MOSIMANN e REIS, 1975/1976; REITZ et al., 1983). A espécie é indicada para recuperação de ecossistemas degradados, particularmente para o reflorestamento de margens de reservatórios de hidrelétricas, por apresentar desenvolvimento rápido, além de produzir frutos muito procurados por pássaros e animais (REITZ et al.,1983; GLUFKE, 1999).

A propagação vegetativa é indicada para plantas que não se reproduzem bem por processos sexuados, que não transmitem por via hereditária determinadas características, como forma da planta, variantes na cor das flores ou aspectos peculiares, assim como quando há uma pequena quantidade de sementes formadas ou quando estas perdem rapidamente seu poder germinativo (KRAMER e KOSLOWSKI, 1960). Com isso, abrevia-se o tempo da cultura e obtém-se floração precoce, pois mudas de plantas adultas, provenientes de propagação vegetativa, são capazes de florescer mais rapidamente que aquelas originadas por sementes, uma vez que não passam pela fase de plântula, já sendo fisiologicamente juvenis, característica muito interessante para a recuperação de ecossistemas degradados (HEROLD, 1982; HARTMANN et al., 2002).

A produção de mudas de araticum-de-porco via sementes apresenta dificuldades, como ocorre em outras anonáceas nativas ou cultivadas. Isto é devido à combinação decorrente da polinização entre as várias espécies desta família, entre dormência fisiológica (decorrente de embrião imaturo) e dormência tegumentar. Assim, juntamente com o estudo sobre a tecnologia de suas sementes, a propagação assexuada é um caminho lógico para possibilitar a produção de mudas.

São muitos os fatores que podem influenciar o enraizamento de estacas, tais como condição fisiológica da planta-mãe, juvenilidade/idade da plantamãe, condição fisiológica das estacas, cofatores do enraizamento, inibidores naturais, época do ano, concentração ideal de fitorreguladores, condições ambientais durante o enraizamento e substrato a ser utilizado (HARTMANN et al., 2002). Como primeiro passo para elaboração de um protocolo de produção de mudas via estaquia, o presente trabalho avaliou épocas de obtenção de estacas e concentrações de auxinas para o enraizamento.

\section{METODOLOGIA}

O experimento foi instalado em casa-devegetação pertencente à Embrapa Florestas, em Colombo - PR (bioma Floresta Ombrófila Mista), nas quatro estações do ano: outono (abril/2000), inverno (julho/2000), primavera (outubro/2000), verão (janeiro/ 2001). As instalações foram realizadas no segundo mês de cada estação, a fim de se obter uma maior representatividade das variações ambientais sofridas pelas plantas-matrizes no campo.

Das copas de árvores-matrizes adultas, localizadas em Colombo - PR, foram colhidos, no período da manhã, ramos semilenhosos provindos de brotações do ano. Foram confeccionadas estacas com tamanho de $10 \mathrm{~cm}$, cortadas em bisel na base, com duas folhas no ápice, cortadas pela metade a fim de reduzir a superfície de transpiração.

As estacas foram submetidas à desinfestação em hipoclorito de sódio $0,5 \%$, por 5 minutos, permanecendo o mesmo período em água corrente, para retirada do excesso do produto. Posteriormente, as bases das estacas permaneceram imersas por 15 minutos em solução de fungicida sistêmico Benlate $\mathbb{}$ (benomyl) 0,5\%,sendo em seguida imersas por 10 segundos nas soluções de ácido indol butírico (AIB) e ácido naftaleno acético (ANA) conforme segue:

T1 - Testemunha (água destilada)
T2 - AIB $2000 \mathrm{mg} \mathrm{L}^{-1}$
T3 - AIB $4000 \mathrm{mg} \mathrm{L}^{-1}$
T4 - AIB $6000 \mathrm{mg} \mathrm{L}^{-1}$
T5 - ANA $2000 \mathrm{mg} \mathrm{L}^{-1}$
T6 - ANA $4000 \mathrm{mg} \mathrm{L}^{-1}$
T7 - ANA $6000 \mathrm{mg} \mathrm{L}^{-1}$
T8 - AIB $2000 \mathrm{mg} \mathrm{L}^{-1}+$ ANA $2000 \mathrm{mg} \mathrm{L}^{-1}$
T9 - ANA $5000 \mathrm{mg} \mathrm{L}^{-1}$ (Raizon $05 \AA$ - produto comercial do Laboratório Okochi Ltda., aplicado na forma de talco)

As estacas foram plantadas em tubetes com vermiculita de granulação média e acondicionados em casa-de-vegetação com sistema de nebulização programado para atuar por 5 segundos a cada 10 minutos. Quinzenalmente, foram realizadas adubações foliares com o adubo líquido do Laboratório Boutin ( $\mathrm{N}$ total: $16 \%$, P solúvel e CNA + água: $4 \%, \mathrm{~K}$ solúvel: 4\%, S: 0,1\%, B: 0,02\%, Cu: 0,05\%, Zn: 0,05\%, Mn: 0,02\%,Mg: 0,01\%, Mo: 0,0005\%). As avaliações foram realizadas 60 dias após a instalação, pela contagem de estacas enraizadas, com calos, vivas e mortas.

O delineamento experimental utilizado foi o inteiramente casualizado, com 9 tratamentos, 5 repetições e 10 estacas por parcela, perfazendo um total de 50 estacas por tratamento e 450 estacas por estação. Para a análise estatística, as épocas foram consideradas separadamente. A homogeneidade das variâncias dos tratamentos foi analisada pelo teste de "Bartlett". As variáveis cujas variâncias dos tratamentos se mostraram homogêneas tiveram as médias dos tratamentos testadas pelo teste de $\mathrm{F}$; as que 
PINTO, L.S. et al. Indução do enraizamento de estacas...

apresentaram heterogeneidade, ou tratavam de contagem, tiveram seus valores originais transformados tomando-se $(X+10)^{1 / 2}$, e posterior análise dos dados transformados. Quando os resultados da análise de variância revelaram diferenças significativas entre as médias dos tratamentos, eles foram comparados pelo teste de Tukey ao nível de $5 \%$ de probabilidade.

\section{RESULTADOS E DISCUSSÃO}

Nas Tabelas 1, 2, 3 e 4, encontram-se os resultados obtidos com araticum-de-porco para todas as variáveis analisadas. Não houve diferença estatística entre os tratamentos no inverno, primavera e verão
(Tabelas 2, 3 e 4); apenas no outono (Tabela 1) houve diferença em relação às estacas vivas (sem raiz ou calo) e às estacas mortas. As maiores presenças de calos $\mathrm{e}$ de estacas vivas na primavera indicam que esta época é, potencialmente, a mais favorável para a estaquia de araticum-de-porco.

Para esta espécie, a elevada mortalidade na época do outono $(83,1 \%)$, provavelmente deveu-se à condição fisiológica das estacas (Tabela 1). Outros fatores podem estar envolvidos na mortalidade de araticum-de-porco, tais como variabilidade genética do material utilizado e programação inadequada da nebulização

na casa-de-vegetação, podendo ter havido um excesso de água para esta espécie, vindo a causar o apodrecimento de muitas estacas.

TABELA 1 - Resultados das médias e desvio padrão para porcentagem de estacas de araticum-de-porco enraizadas, com calos, sem raiz ou calo e mortas, entre os tratamentos, coletadas em abril de 2000 (outono).

\begin{tabular}{|c|c|c|c|c|}
\hline \multirow[t]{2}{*}{ Tratamentos } & \multicolumn{3}{|c|}{ Estacas vivas } & \multirow{2}{*}{$\begin{array}{c}\text { Estacas } \\
\text { mortas } \\
(\%)\end{array}$} \\
\hline & $\begin{array}{c}\text { Enraizadas } \\
(\%)\end{array}$ & $\begin{array}{c}\text { Com calos } \\
(\%)\end{array}$ & $\begin{array}{c}\text { Sem raiz ou calo } \\
(\%)\end{array}$ & \\
\hline T1- Testemunha & $0 \mathrm{a}$ & $0 \mathrm{a}$ & $16 \pm 16,7 \mathrm{abc}$ & $84 \pm 16,7 \mathrm{abc}$ \\
\hline T2- AIB $2000 \mathrm{mg} \cdot \mathrm{L}^{-1}$ & $0 \mathrm{a}$ & $0 \mathrm{a}$ & $10 \pm 10 \quad b c$ & $90 \pm 10 \quad a b$ \\
\hline T3- AIB $4000 \mathrm{mg} \cdot \mathrm{L}^{-1}$ & $0 \mathrm{a}$ & $0 \mathrm{a}$ & $6 \pm \overline{5}, 5$ & $94 \pm 5,5$ \\
\hline T4- AIB $6000 \mathrm{mg} \cdot \mathrm{L}^{-1}$ & $0 \mathrm{a}$ & $2 a$ & $32+8,4 \quad a b$ & $66 \pm 8,9$ \\
\hline T5- ANA $2000 \mathrm{mg} \cdot \mathrm{L}^{-1}$ & $0 \mathrm{a}$ & $0 \mathrm{a}$ & $16+11,4 a b c$ & $84+11,4 a b c$ \\
\hline T6- ANA 4000 mg.L ${ }^{-1}$ & $0 \mathrm{a}$ & $0 \mathrm{a}$ & $10 \pm 14,1 \quad b c$ & $90 \pm 14,1 \quad a b$ \\
\hline T7- ANA $6000 \mathrm{mg} \cdot \mathrm{L}^{-1}$ & $0 \mathrm{a}$ & $0 \mathrm{a}$ & $14 \pm 8,9 \quad a b c$ & $86 \pm 8,9 a b c$ \\
\hline T8- AIB $2000 \mathrm{mg} \cdot \mathrm{L}^{-1}+$ ANA $2000 \mathrm{mg} \cdot \mathrm{L}^{-1}$ & $0 \mathrm{a}$ & $0 \mathrm{a}$ & $38 \pm 14,8 \mathrm{abc}$ & $62 \pm 14,8 \quad c$ \\
\hline T9- ANA $5000 \mathrm{mg} \cdot \mathrm{L}^{-1}$ & $0 \mathrm{a}$ & $0 \mathrm{a}$ & $8+11 \quad b c$ & $92+11$ \\
\hline Média geral & 0,7 & 0,2 & $16,7+14,9$ & $83,1+15,2$ \\
\hline Coeficiente de Variação & - & - & 70,14 & 14,12 \\
\hline
\end{tabular}

Médias acompanhadas de mesma letra na coluna não diferem ao nível de $5 \%$ de probabilidade pelo Teste de Tukey.

TABELA 2 - Resultados das médias e desvio padrão para porcentagem de estacas de araticum-de-porco enraizadas, com calos, sem raiz ou calo e mortas, entre os tratamentos, coletadas em julho de 2000 (inverno).

\begin{tabular}{|c|c|c|c|c|}
\hline \multirow[t]{2}{*}{ Tratamentos } & \multicolumn{3}{|c|}{ Estacas vivas } & \multirow{2}{*}{$\begin{array}{c}\text { Estacas } \\
\text { mortas } \\
(\%)\end{array}$} \\
\hline & $\begin{array}{c}\text { Enraizadas } \\
(\%)\end{array}$ & $\begin{array}{c}\text { Com calos } \\
(\%)\end{array}$ & $\begin{array}{c}\text { Sem raiz ou calo } \\
(\%)\end{array}$ & \\
\hline T1- Testemunha & $0 a$ & $0 a$ & $72 \pm 14,8 a$ & $28 \pm 14,8 a$ \\
\hline T2- AIB $2000 \mathrm{mg} \cdot \mathrm{L}^{-1}$ & $0 \mathrm{a}$ & $0 \mathrm{a}$ & $58 \pm 23,9 a$ & $43 \pm 23,9 a$ \\
\hline T3- AIB $4000 \mathrm{mg} \cdot \mathrm{L}^{-1}$ & $0 \mathrm{a}$ & $0 \mathrm{a}$ & $64 \pm 13,4 a$ & $36 \pm 13,4 a$ \\
\hline T4- AIB $6000 \mathrm{mg} \cdot \mathrm{L}^{-1}$ & $0 \mathrm{a}$ & $2 a$ & $62 \pm 14,8 a$ & $36 \pm 15,2 a$ \\
\hline T5- ANA 2000 mg. $\mathrm{L}^{-1}$ & $0 \mathrm{a}$ & $4 \mathrm{a}$ & $62 \pm 13,0 a$ & $34 \pm 18,2 a$ \\
\hline T6- ANA $4000 \mathrm{mg} \cdot \mathrm{L}^{-1}$ & $0 \mathrm{a}$ & $0 \mathrm{a}$ & $62 \pm 22,8 a$ & $38 \pm 22,8 a$ \\
\hline T7- ANA $6000 \mathrm{mg}^{-\mathrm{L}^{-1}}$ & $0 \mathrm{a}$ & $4 a$ & $68 \pm 17,9 a$ & $28+21,7 a$ \\
\hline T8- AIB $2000 \mathrm{mg} \cdot \mathrm{L}^{-1}+$ ANA $2000 \mathrm{mg} \cdot \mathrm{L}^{-1}$ & $0 \mathrm{a}$ & $2 a$ & $50 \pm 28,3 a$ & $48 \pm 31,9 a$ \\
\hline T9- ANA $5000 \mathrm{mg} \cdot \mathrm{L}^{-1}$ & $0 \mathrm{a}$ & $4 a$ & $70 \pm 7,1$ a & $26 \pm 5,5 a$ \\
\hline Média geral & 0 & 1,3 & $63,1 \pm 17,8$ & $35,1 \pm 19,3$ \\
\hline Coeficiente de Variação & - & - & $2 \overline{9}, 17$ & $5 \overline{6}, 72$ \\
\hline
\end{tabular}

Médias acompanhadas de mesma letra na coluna não diferem ao nível de $5 \%$ de probabilidade pelo Teste de Tukey. 
PINTO, L.S. et al. Indução do enraizamento de estacas...

É fato comprovado que estacas da parte aérea de anonáceas são consideradas de difícil enraizamento (GETTYS et al., 1995). Assim, os resultados concordam com investigações sobre outras anonáceas já cultivadas pelo valor de seus frutos. Em um estudo com estacas enfolhadas de graviola (Annona muricata L.), tratadas com diversos fitorreguladores, não foi obtido nenhum enraizamento (CASAS et al., 1984). Bankar (1989) teve, como melhor resultado de Annona squamosa, $26 \%$ de enraizamento com AIB 2500 - $3000 \mathrm{mg} \cdot \mathrm{L}^{-1}$, demonstrando a necessidade do uso de fitorreguladores, pelo fato da testemunha ter apresentado somente $4 \%$ de enraizamento. Ferreira e Cereda (1999) obtiveram $27 \%$ de enraizamento de atemóia utilizando fitorregulador Rootone ${ }^{\circledR}$ e $25 \%$ com a testemunha, resultados, que conforme os autores, são altos em se tratando de estacas de difícil enraizamento.

TABELA 3 - Resultados das médias e desvio padrão para porcentagem de estacas de araticum-de-porco enraizadas, com calos, sem raiz ou calo e mortas, entre os tratamentos, coletadas em outubro de 2000 (primavera).

\begin{tabular}{|c|c|c|c|c|}
\hline \multirow[t]{2}{*}{ Tratamentos } & \multicolumn{3}{|c|}{ Estavas vivas } & \multirow{2}{*}{$\begin{array}{c}\text { Estacas } \\
\text { mortas } \\
(\%)\end{array}$} \\
\hline & $\begin{array}{c}\text { Enraizadas } \\
(\%)\end{array}$ & $\begin{array}{c}\text { Com calos } \\
(\%)\end{array}$ & $\begin{array}{l}\text { Sem raiz ou } \\
\text { calo }(\%)\end{array}$ & \\
\hline T1- Testemunha & $0 a$ & $24 \pm 11,4 a$ & $36 \pm 18,2 a$ & $40 \pm 27,4 a$ \\
\hline T2- AIB $2000 \mathrm{mg} \cdot \mathrm{L}^{-1}$ & $0 \mathrm{a}$ & $30 \pm 7,1$ a & $46 \pm 19,5$ a & $24 \pm 13,4 a$ \\
\hline T3- AIB $4000 \mathrm{mg} \cdot \mathrm{L}^{-1}$ & $0 \mathrm{a}$ & $36 \pm 15,2 a$ & $36 \pm 16,7$ a & $28 \pm 19,2 \mathrm{a}$ \\
\hline T4- AIB $6000 \mathrm{mg} \cdot \mathrm{L}^{-1}$ & $0 \mathrm{a}$ & $36 \pm 15,2 a$ & $44 \pm 28,8 a$ & $20 \pm 20,0 a$ \\
\hline T5- ANA $2000 \mathrm{mg} \cdot \mathrm{L}^{-1}$ & $0 \mathrm{a}$ & $14 \pm 5,5$ a & $28 \pm 13,0$ a & $58 \pm 8,4$ a \\
\hline T6- ANA $4000 \mathrm{mg}^{-\mathrm{L}^{-1}}$ & $2 a$ & $30 \pm 12,2 a$ & $40 \pm 18,7 \mathrm{a}$ & $28 \pm 13,0 a$ \\
\hline T7- ANA $6000 \mathrm{mg} \cdot \mathrm{L}^{-1}$ & $4 a$ & $24+18,2 \mathrm{a}$ & $34 \pm 26,1 \mathrm{a}$ & $38+33,0 a$ \\
\hline T8- AIB $2000 \mathrm{mg} \cdot \mathrm{L}^{-1}$ + ANA $2000 \mathrm{mg} \cdot \mathrm{L}^{-1}$ & $4 a$ & $32 \pm 19,2 \mathrm{a}$ & $30 \pm 25,5 a$ & $34 \pm 19,5 a$ \\
\hline T9- ANA $5000 \mathrm{mg} \cdot \mathrm{L}^{-1}$ & $0 \mathrm{a}$ & $32+13,0$ a & $34+15,2 a$ & $34+11,4 a$ \\
\hline Média geral & 1,1 & $28, \overline{7} \pm 14,1$ & $36,4 \pm 19,7$ & $33, \overline{8} \pm 18,7$ \\
\hline Coeficiente de Variação & - & $4 \overline{7,80}$ & $5 \overline{7,12}$ & 50,51 \\
\hline
\end{tabular}

Médias acompanhadas de mesma letra na coluna não diferem ao nível de $5 \%$ de probabilidade pelo Teste de Tukey.

TABELA4 - Resultados das médias e desvio padrão para porcentagem de estacas de araticum-de-porco enraizadas, com calos, sem raiz ou calo e mortas, entre os tratamentos, coletadas em fevereiro de 2001 (verão).

\begin{tabular}{|c|c|c|c|c|}
\hline \multirow[b]{2}{*}{ Tratamentos } & \multicolumn{3}{|c|}{ Estacas vivas } & \multirow{2}{*}{$\begin{array}{c}\text { Estacas } \\
\text { mortas } \\
(\%)\end{array}$} \\
\hline & $\begin{array}{c}\text { Enraizadas } \\
(\%)\end{array}$ & $\begin{array}{c}\text { Com calos } \\
(\%)\end{array}$ & $\begin{array}{c}\text { Sem raiz ou calo } \\
(\%)\end{array}$ & \\
\hline T1- Testemunha & $2 a$ & $16 \pm 15,2 a$ & $16 \pm 13,4 a$ & $66 \pm 20,7 a$ \\
\hline T2- AIB $2000 \mathrm{mg} \cdot \mathrm{L}^{-1}$ & $0 \mathrm{a}$ & $10 \pm 14,1 \mathrm{a}$ & $12 \pm 11,0 a$ & $78 \pm 21,7 a$ \\
\hline T3- AIB $4000 \mathrm{mg} \cdot \mathrm{L}^{-1}$ & $2 a$ & $10 \pm 12,2 \mathrm{a}$ & $22 \pm 11,0 a$ & $66 \pm 20,7 a$ \\
\hline T4- AIB $6000 \mathrm{mg} \cdot \mathrm{L}^{-1}$ & $0 \mathrm{a}$ & $12 \pm 13,0 \mathrm{a}$ & $16 \pm 19,5 a$ & $72 \pm 23,9 a$ \\
\hline T5- ANA $2000 \mathrm{mg} \cdot \mathrm{L}^{-1}$ & $0 \mathrm{a}$ & $14 \mp 13,4 a$ & $18 \mp 16,4 a$ & $68 \mp 29,5$ \\
\hline T6- ANA $4000 \mathrm{mg} \cdot \mathrm{L}^{-1}$ & $0 \mathrm{a}$ & $16+13,4 a$ & $32+8,4$ a & $52+14,8 a$ \\
\hline T7- ANA 6000 mg.L ${ }^{-1}$ & $2 a$ & $22+17,9 a$ & $30+7,1$ a & $46+24,1 \mathrm{a}$ \\
\hline T8- AIB $2000 \mathrm{mg} \cdot \mathrm{L}^{-1}$ + ANA $2000 \mathrm{mg} \cdot \mathrm{L}^{-1}$ & $0 \mathrm{a}$ & $06 \pm 13,4 a$ & $18 \pm 11,0 \mathrm{a}$ & $76 \pm 18,2 \mathrm{a}$ \\
\hline T9- ANA 5000 mg.L ${ }^{-1}$ & $0 \mathrm{a}$ & $04 \pm 5,5$ a & $14 \pm 13,4 a$ & $82 \pm 17,9 a$ \\
\hline Média geral & 0,7 & $12,2+13,3$ & $19, \overline{8}+13,4$ & $67, \overline{3}+22,6$ \\
\hline Coeficiente de Variação & - & 110,45 & 65,06 & 32,16 \\
\hline
\end{tabular}

Médias acompanhadas de mesma letra na coluna não diferem ao nível de $5 \%$ de probabilidade pelo Teste de Tukey. 
PINTO, L.S. et al. Indução do enraizamento de estacas...

Na natureza, após distúrbios ambientais como queimadas, Rollinia rugulosa e várias outras anonáceas nativas apresentam propagação vegetativa a partir de brotações de raízes ou de caules subterrâneos, também chamados de sóboles (RIZZINI e HERINGER, 1962; RIZZINI, 1973; ZÁCHIA, 1994). Essas brotações provenientes de raízes ou de caules, que ocorrem na base das plantas-matrizes em condições naturais, bem como brotações jovens de caules, oriundas de rebrota, constituem um material muito promissor para a estaquia, uma vez que se trata de material jovem ou rejuvenescido (HARTMANN et al., 2002) e seu uso racional, que requer obtenção controlada das estacas, poderá melhorar o desempenho da estaquia em espécies classificadas como difíceis de enraizar, como parece ser o caso da Rollinia rugulosa.

\section{CONCLUSÃO}

Estacas da copa de árvores de Rollinia rugulosa têm capacidade muito baixa de produção de raízes em qualquer época do ano, mesmo quando estimuladas com o uso de fitorreguladores nas condições e concentrações utilizadas no presente trabalho.

\section{AGRADECIMENTOS}

Aos funcionários da Embrapa Florestas, Srs. Vero Oscar Cardoso dos Santos, Carlos Amilcar de Carvalho Silva e Paulino Graff, pela ajuda constante durante o experimento.

\section{REFERÊNCIAS}

1. BBANKAR, G. J. Vegetative propagation in annonas (Annona squamosa L.). Journal of Horticultural Science, Ashford, v. 18, n. 1-2, p. 10-13, 1989.

2. CASAS, M. H.; VICTÓRIA, S. M. A.; ZARATE, R. R. D. Preliminary trials on sexual and assexual propagation of soursop (Annona muricata), Palmira, v. 4, p. 66-81, 1984.

3. FERREIRA, G.; CEREDA, E. Efeito da interação entre fitorreguladores, substratos e tipos de estacas no enraizamento de atemóia (Annona cherimolia Mill. x A. squamosa L.). Revista Brasileira de Fruticultura, Jaboticabal, v. 21, n. 1, p. 79-83, 1999.

4. GETTYS, L.; DUKE, E.; COX, A. Vegetative propagation of a native pawpaw - Asimina tetramera. In: ANNUAL MEETING OF THE FLORIDA STATE HORTICULTURAL SOCIETY, 108., 1995, Orlando. Proceedings. Orlando: 1995, p. 389-391.

5. GLUFKE, C. Espécies florestais recomendadas para recuperação de áreas degradadas. Porto Alegre: Fundação Zoobotânica do Rio Grande do Sul, 1999. 48p.

6. HARTMANN, H. T.; KESTER, D.E.; DAVIES JR, F. T.; GENEVE, R. L. Plant propagation: principles and practices. 7 ed. Nova Jersey: Prentice Hall, 2002. 880p.

7. HEROLD, H. Como cultivar cactos. Rio de Janeiro: Tecnoprint, 1982. 121p.

8. KRAMER, P.J.; KOZLOWSKI, T.T. Fisiologia das árvores. 1 ed. Lisboa: Fundação Calouste Gulbenkian, 1960. 745p.

9. MOSIMANN, R.M.S.; REIS, A. Frutos nativos da ilha de Santa Catarina (Florianópolis). Ínsula, Florianópolis, n.8, p.29-46, 1975/1976.

10. REITZ, P. R. ; KLEIN, R. M. ; REIS, A. Projeto Madeira do Rio Grande do Sul. Sellowia, Itajaí, v. 34-35, p. 1-525, 1983.

11. RIZZINI, C.T. Dormancy in seeds of Anona crassiflora Mart. Journal of Experimental Botany, v.24, n. 78, p.117-123, 1973.

12. RIZZINI, C. T.; HERINGER, E.P. Studies on the underground organs of trees and shrubs from some southern Brazilian savannas. Anais da Academia Brasileira de Ciências, Rio de Janeiro, v.34, n.2, p. 235-247, 1962.

13. ZÁCHIA, R.A. Estudos taxonômicos na família Annonaceae Juss. no Rio Grande do Sul. 1994. 366p. (Mestrado em Botânica - Instituto de Biociências, Universidade Federal do Rio Grande do Sul, Porto Alegre). 